\title{
Bushfire Emergency Response Uncertainty Quantification
}

\author{
T. Bruggemann ${ }^{\mathrm{a}}{ }$, J.J. Ford ${ }^{\mathrm{a}}$, G. White ${ }^{\mathrm{a}}{ }$, T. Perez ${ }^{\mathrm{a}}{ }^{\text {, W. Power }}{ }^{\mathrm{b}}$ \\ ${ }^{a}$ Science and Engineering Faculty, Queensland University of Technology, Australia \\ ${ }^{\mathrm{b}}$ Weapons and Combat Systems Division, Defence Science \& Technology Group, Australia. \\ Email: t.bruggemann@qut.edu.au
}

\begin{abstract}
Today, scenarios based on computer simulations are a fundamental tool for informing decision making at different levels of an organisation. When decisions concern the potential behaviour of complex systems, then uncertainty quantification (UQ) is paramount to provide appropriate levels of information for effective decision making. This is especially true for the Department of Defence which needs to make decisions on highly complex systems of systems, in uncertain future scenarios. However, it is useful to explore the tools and techniques required to analyse and communicate this uncertainty in an unclassified manner. Therefore, this project developed an unclassified, bushfire emergency response simulation to understand how to model, manage and communicate the impact of uncertainty in complex systems, as a surrogate for similar Defence problems. In this scenario, the fire is a threat and can exhibit behaviours characteristic of a complex system. The fire interacts with a network of response unit models, which in response to the complex behaviour of the fire, exhibit complex and uncertain behaviour, all while following mostly simple, deterministic logic. These models are comprised of the assets to be protected, command and control assets, fixed and mobile sensing assets, and assets within the response team with different capabilities to move and fight the fire (Fig. 1). The simulator is intended to capture key emergency response dynamic characteristics.
\end{abstract}

This is the first in a series of papers utilising bushfire emergency response simulation as a surrogate for Defence problems to explore the quantification of uncertainty in modelling, simulation and analysis of complex systems. This paper addresses the effects of input and output uncertainty, while future papers will address other sources of uncertainty, such as uncertainty in the operational environment, mission, agent behaviour, and importantly the communication of the impact of these var-

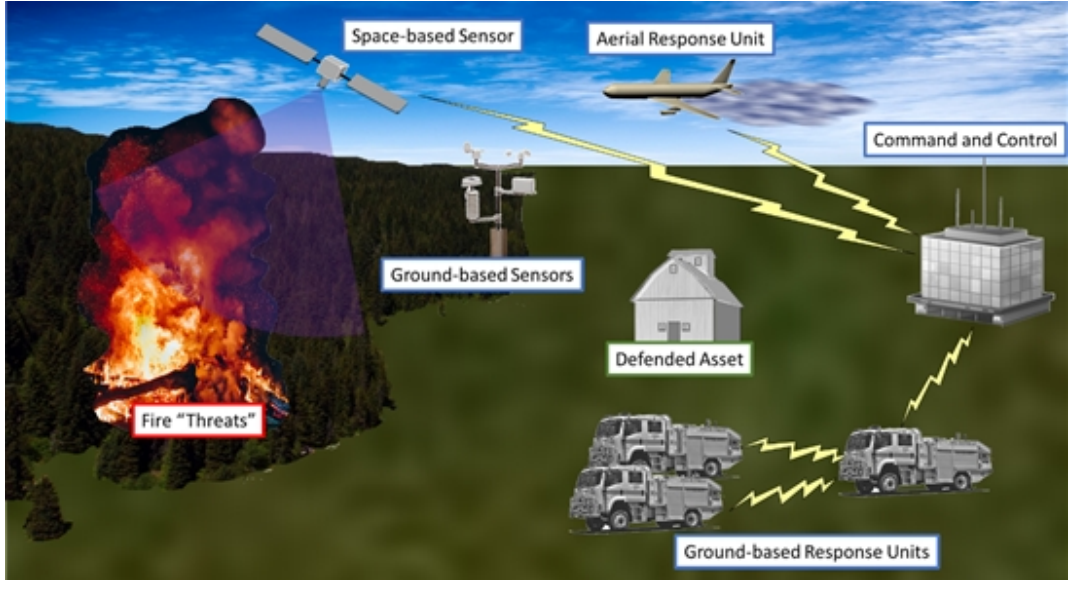

Figure 1. Simplified operational context diagram of bushfire emergency response. ious sources of uncertainty to decision makers.

Keywords: Uncertainty quantification, bushfire emergency response, Monte Carlo simulation 


\section{INTRODUCTION}

As Defence systems become increasingly complex, the Australian Department of Defence needs new tools to model, manage and communicate the impact of uncertainty in complex dynamical systems modelling, simulations and analysis. Exploring the tools and techniques required to treat this uncertainty in an unclassified manner is useful. Quantifying uncertainty in different situations and analysing how uncertainties interact is an important problem for bushfire emergency response when making decisions about developing and shaping future capability. For this project, bushfire emergency response was chosen as an unclassified surrogate for a range of Defence problems.

Bushfire behaviour occurs over different spatio-temporal scales, making it difficult to predict the rate of spread, given numerous complex variables such as fuel, wind and terrain (Miller et al. (2015)). Bushfire simulators model a bushfire's spatial and temporal dynamics in different scenarios and are used to aid the prediction and management of bushfires (Miller et al. (2015); Sullivan (2009)). State of the art examples include PHOENIX Rapidfire (Tolhurst et al. (2008)), AUSTRALIS (Johnston et al. (2008)), and (SPARK Miller et al. (2015)). These simulators include comprehensive environment, fire behaviour, propagation, fuel, and asset impact models enabling predictions, statistics and visualization of bushfire spread to aid decision makers. Systems used to quantify uncertainty in bushfire response include FireDST (French et al. (2013)) and SABRE (Twomey and Sturgess (2016)) which are PHOENIX-based integrated fire risk assessment tools. These use a deterministic simulation model together with probabilities to perform the risk assessment and provide decision support. In contrast, this paper focuses on a general framework and methodology to characterizing the impact of uncertainty in complex systems, as a surrogate for similar Defence problems. For this purpose, our framework considers the dynamical interactions between a fire fighting team, support assets and a bushfire threat, enabling simulation and analysis of different operational, tactical and strategic management decisions.

Table 1 describes an incomplete list Table 1. Sources of Uncertainty for BERS Modelling. of example sources of uncertainty for $\quad$ Nature of Uncertainty $($ Smith $i \overline{2013}$ ) the Bushfire Emergency Response Scenario (BERS) modelling, simulation and analysis activity. These uncertainties are categorised by the "nature" of the uncertainty as described in (Smith

\begin{tabular}{|c|c|}
\hline Nature of Uncertainty $\mathbf{i}$ Smith $\mathbf{i 2 1 3}$ & Key Uncertainty Sources \\
\hline Experimental uncertainties / limitations & $\begin{array}{c}\text { Deployment scenario / mission } \\
\text { System performance / data }\end{array}$ \\
\hline Model errors / discrepancies & System functionality \\
& Decision making (human or machine) \\
\hline Input uncertainties & Natural environment \\
& Threat behaviour \\
\hline
\end{tabular}
(2013)). Another source of uncertainty is "numerical errors and uncertainties" in the computation of the simulation results, but these are "the least uncertain component of predictive sciences" (Smith (2013)) and are not described in the table. Smith (2013) presents a larger framework for uncertainty quantification (UQ).

In addition to fire behaviour uncertainty, there is also uncertainty in the response to bushfire emergencies, including response team design, technology, equipment, operational deployment, human factors and the outcomes of these decisions. For example, how can decision makers be confident that they have prepared the right number and type of vehicles and equipment, and how successful they will be? There are few tools available to decision makers for quantifying these kinds of uncertainties. Emergency response simulation is complicated by the necessity to perform many computationally intensive simulations and the lack of knowledge regarding uncertain inputs and outputs. This paper presents a simulation methodology that simplifies the process of uncertainty analysis and quantifies the uncertainty that arises in complex systems.

\section{MISSION AND OPERATIONAL ENVIRONMENT AND UNCERTAINTY}

The mission definition includes defining the operational environment, the bushfire threat, and the response teams. A Bushfire Emergency Response Scenario (BERS) with response team assets is depicted in Fig. 1. The mission is considered to include a Bushfire Threat (BFT) which are spot bushfires occurring at any location within the considered area of operations. Defended Assets (DA) are stationary ground-based assets that are vulnerable to the BFT. The Bushfire Response Team (BRT) includes a Global Command and Control Unit (GC2), which is a global command and control (C2) unit that sets the BFT response strategy and provides execution plans for the different response teams and units. Local Response C2 Units (LC2s) are mobile local $\mathrm{C} 2$ units that communicate with their associated fire-fighting response units and the global $\mathrm{C} 2$ unit. These units set the local BFT strategy and provide execution plans for their assigned ground-based response units at the local level. Ground-based Response Units (GRUs) are mobile units with local fire-sensing as well as local low-power fire-fighting capabilities. GRUs can communicate with LC2 to which they are assigned. Aerial-response Units (ARUs) have sensing capability as well as high-power capability to extinguish the fire. 
Ground-based Sensing Units (GSU) are spatially distributed sensors over the area of operations. Space-based Sensing Units (SSU) are satellite-like sensors that can provide global information related to the state of the BFT and response team at specific times.

\section{MODELLING AND SIMULATION FOR UNCERTAINTY QUANTIFICATION (UQ)}

Fig. 2 shows the UQ work flow. A UQ definition process is required to define the mission and UQ objectives, operational environment, system behaviours, and performance hypotheses which are propositions about the mission and objectives to be tested, based on data generated from the simulation scenarios and available background information.

The simulation for the UQ involves setting up the simulator, performing UQ of the inputs and generating input data, followed by running the simulation and generating output data to perform the UQ of the Mission. The outcomes of the analysis can then be used to communicate to decision makers about operational strategies, or BRT design, while described within the appropriate context as defined by the UQ.

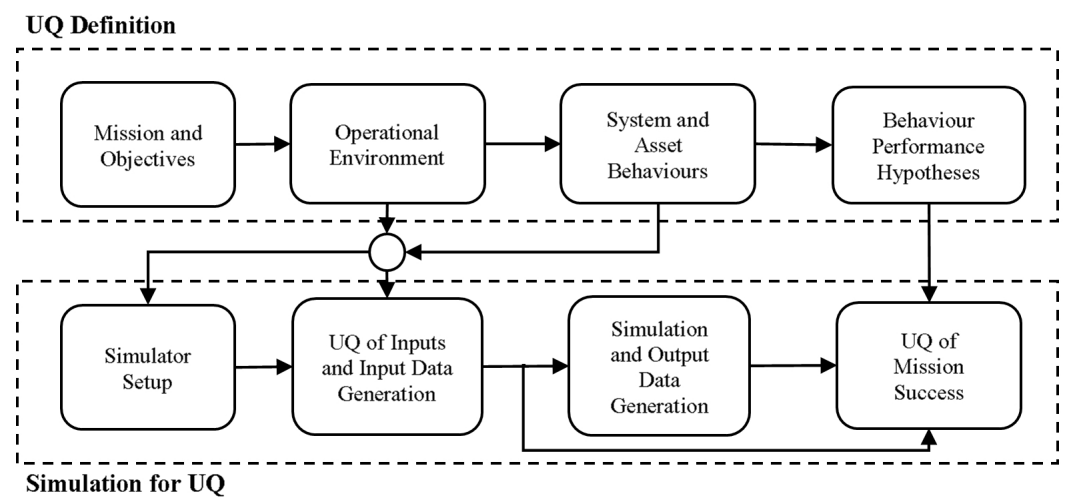

Figure 2. Simulation methodology.

\section{SIMULATION OBJECTIVES AND PERFORMANCE HYPOTHESES}

It is often desired to analyse the performance of the BRT given a number of fires and assets with a range of fire behaviours and initial conditions such as location, in the presence of uncertainty. A performance hypothesis is considered to be $O:\{$ Mission success is achieved under the required operational conditions $\}$ and then its probability is evaluated conditioned on propositions related to available background information $I$ and the data $D$ from the simulation scenarios, namely, $P_{r}(O \mid D, I)$. For example, two mission success measures might be: (1) Keep all threats out of pre-defined range of a DA and (2) Avoid loss (destruction) of fire-fighting assets.

\section{SOURCES OF UNCERTAINTY IN BERS MODELLING, SIMULATION AND ANALYSIS}

In BERS UQ, we are concerned with simulating models of the form:

$$
\begin{aligned}
\boldsymbol{x}_{t+1} & =\boldsymbol{f}\left(t, \boldsymbol{x}_{t}, \boldsymbol{u}_{t}, \boldsymbol{\theta}\right)+\boldsymbol{\eta}_{t}, \\
\boldsymbol{y}_{t} & =\boldsymbol{g}\left(t, \boldsymbol{x}_{t}, \boldsymbol{u}_{t}, \boldsymbol{\theta}\right)+\boldsymbol{\nu}_{t},
\end{aligned}
$$

where $\boldsymbol{x}_{t}$ is the state of the BERS system, with initial state $\boldsymbol{x}_{t=0}=\boldsymbol{x}_{0} ; \boldsymbol{u}_{t}$ is the exogenous input to the system; $\boldsymbol{\theta}$ is the vector of model parameters; $\boldsymbol{y}_{t}$ is the output or observed attributes of the system; $\boldsymbol{f}$ is the state (transition) model and $\boldsymbol{g}$ is the output (measurement) model. $\boldsymbol{\eta}_{t}$ and $\boldsymbol{\nu}_{t}$ are random variables assumed to be following a Gaussian density function conditioned on prior information $I$. $\boldsymbol{\eta}_{t}$ represents the error (assumed additive) with which the model $\boldsymbol{f}$ describes the transition from the current state $\boldsymbol{x}_{t}$ to the successor state $\boldsymbol{x}_{t+1}$. Similarly, $\boldsymbol{\nu}_{t}$ represents the error (assumed additive) with which the model $\boldsymbol{g}$ describes the output. The most fundamental question in UQ for simulation models is not how to propagate uncertainty through the simulator, but how to model the uncertainty associated with the inputs that go into the simulator. One possibility is to use the maximum entropy principle approach to characterise the input uncertainty (Jaynes (1957)). For example,the maximum entropy (MaxEnt) distribution is the uniform distribution when $p(x)=1 /(b-a)$ for $a \leq x \leq b$, the Laplace probability density when $x$ takes values in $(-\infty, \infty)$, and the exponential probability density when $x$ takes values in $[0, \infty)$ (Kapur (1989)).

In order to simulate a scenario we need to draw realisations of $p(\boldsymbol{\theta} \mid I), p\left(\boldsymbol{x}_{0} \mid I\right), p_{\eta}\left(\boldsymbol{\eta}_{t} \mid I\right), p_{\nu}\left(\boldsymbol{\nu}_{t} \mid I\right)$ in order to initialise the simulator. The theory of sampling is well developed and will not be reviewed here (Bolstad (2010). Kroese et al. (2011)). The following sources of uncertainty can be identified:

- Input uncertainty includes parametric uncertainty which is uncertainty in $\boldsymbol{\theta}$, and initial-condition uncertainty which is uncertainty in the value of the state at time zero. 
- Model uncertainty is uncertainty in the structure of the state and measurement functions $\boldsymbol{f}$ and $\boldsymbol{g}$ including the equations themselves, the algorithms used to implement the equations in simulation, and the coupling of the models (Freni and Mannina (2010)).

- Numerical simulation uncertainty is uncertainty due to numerical equation solution methods, discretisation and resultant complex non-linear behaviours.

- Output uncertainty in the quantities of interest, such as asset survival. In this work we assume $\nu_{t}=0$.

- Other sources of uncertainty within the scenario, such as within the operational environment, mission definition, and asset behaviour; initially, this research is focused on the input and output uncertainty.

The type of complex systems we are concerned with have a number of entities that have the capability to interact (called interaction structure) (Willems and Polderman (2013)). Uncertainty can arise from a number of sources such as stochasticity in the system, sub-systems and through the entity interactions such as "emergent behaviour", i.e. behaviour that cannot be foreseen by study of the entities individually (Green (1993)).

The type of entities considered in BERS modelling are dynamical systems or "agents" that have capability for decision making and actuation (energy and physical interaction), sensing (information interaction) and communication (information exchange) capabilities. Actuation can be categorized into acting upon other entities (e.g. engaging fires), the environment or itself. Sensing can be categorized into acquiring information about other entities, the environment, or itself (e.g. resource level, health). Communication can be categorized into transmitting or receiving information about other entities, the environment, or itself (knowledge/intelligence) or as directives to perform some actions (command and control).

\section{SIMULATION AND OUTPUT DATA GENERATION}

Fig. 3 shows the process and inputs and outputs considered for BERS simulation. Our BERS simulator was initialized with a 80x80 cell grid and a single DA was located at 0 cells North and 40 cells East such that the DA was in the centre of the x-axis. Fig. 4 (left) illustrates the simulator inputs for a single scenario. Fire is defined as the temper-

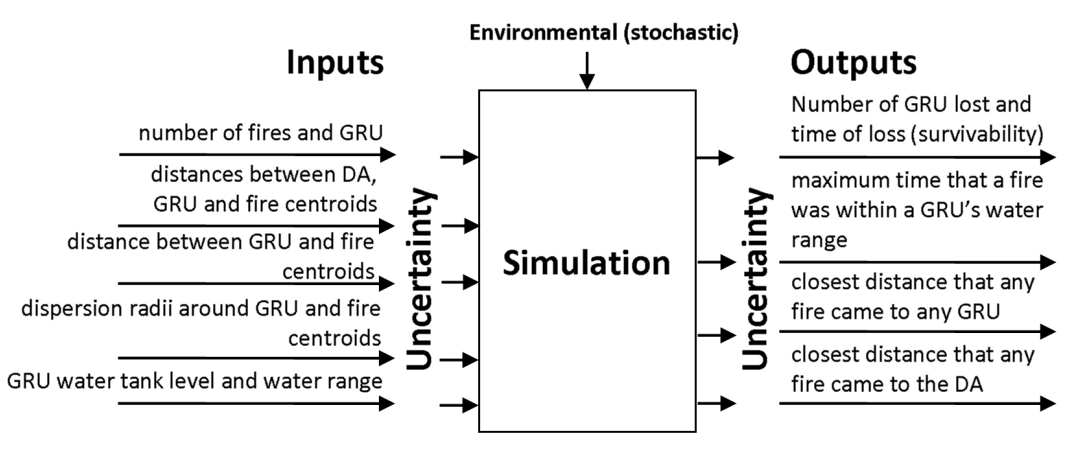

Figure 3. Simulation Process.

ature of a cell as indicated by the cell colour from 0 (blue, coldest), to 1 (red, hottest). Fig. 4 (right) illustrates the closest distance between fire and DA output, near the end of the simulation.

Refer to Bruggemann et al. (2019) for details about the simulator. There are 9 input uncertainty parameters of interest considered, including the number of fire ignition points $\in[1,7]$ and the initial number of GRU $\in[1,10]$. There are 5 parameters for the initial starting location of the fires and GRU which were initialized radially from the DA location. For a given number of GRU, a centroid location was chosen at a random starting distance $\in[0,50]$ cells and angle $\in[-\pi, \pi]$ from the location 0 North and 40 East. Then, individual GRU were dispersed around the centroid by sampling a Gaussian distribution with mean distance $\in[0,10]$ cells and variance of 9 cells. Similarly the fires were initialized at random starting distance $\in[0,50]$ cells and angle $\in[-\pi, \pi]$ from the DA and dispersed around the fire centroid by sampling a Gaussian distribution with mean distance $\in[0,10]$ cells and variance of 9 cells. Two parameters for GRU equipage were considered: GRU water tank level $\in[0,1]$ from 0 (empty) to 1 (full) and GRU water range $\in[5,20]$ cells. There was also noise such as wind speed and direction and environmental parameters that govern fire behaviour (see Bruggemann et al. (2019)). Wind direction was set to vary according to a random walk, constrained to angles that would blow the fire towards the DA, so that fires always posed a threat to the DA. The GRU sensor range was fixed to 20 cells. A GRU was deemed destroyed if the fire above a certain temperature threshold came within 1 cell of the GRU.

The simulator output metrics considered were the number of GRU lost and the time that a GRU was first lost as indicators of survivability; the maximum time that a fire was within a GRU's water range as an indicator of a GRU's fire-fighting effectiveness; the closest distance that any fire came to any GRU as an indicator of 

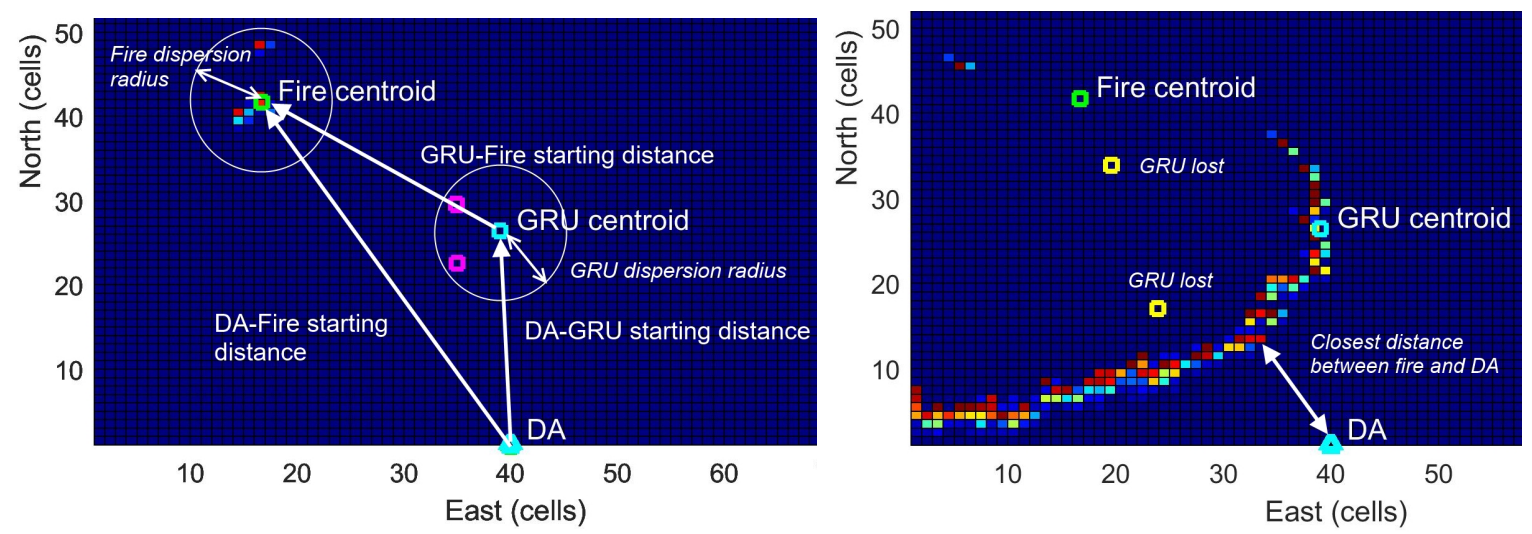

Figure 4. Left: Grid map of the simulation, illustrating the simulator inputs for a single scenario. A single DA (cyan triangle) is located at 0 cells North, 40 cells East. The simulator inputs include the DA to GRU starting distance, DA to Fire starting distance and the GRU to Fire starting distance. From these distances, the location of a GRU centroid (cyan square) and location of a Fire centroid (green square) are determined. Then, individual fire and GRU starting locations are determined from the fire dispersion radius and GRU dispersion radius inputs, respectively. Fire starting locations are shown by the coloured cells around the Fire centroid which indicate cell temperatures from 0 (blue, coldest) to 1 (red, hottest). GRU starting locations for 2 GRU (magenta squares) are shown. Right: Near the end of the simulation, both GRU have been destroyed (at locations indicated by yellow squares), and the fire has formed a front which is moving towards the DA. The closest distance between the fire and DA at this point in simulation is shown.

a GRU's ability to protect itself and; the closest distance that any fire came to the DA as an indicator of the GRU's ability to protect the DA. Only the DA protection and GRU survivability will be considered in the following results.

\section{Results: Uncertainty Quantification About Mission Success}

We consider mission success $S$ as a function of whether performance indices $E_{m}$ are met, which can be either true or false. If we produce $N$ replications (simulations) of a particular operational scenario we will obtain a set of binary data $D$. If $E_{m}$ are Bernoulli distributed then we can find the posterior probability of success $P_{r}(S \mid D)$, numerically from the simulation data (Perez et al. (2012); Perez (2015); Kallinen et al. (2019)).

For example, suppose that success is defined as a defended asset surviving a trial. We assume that the number of times an asset survives $x$, out of $n$ trials, follows a binomial distribution and we wish to calculate the probability of an asset surviving $P_{r}(S)$. Taking a Bayesian approach, we assume that $S$ follows a Bernoulli distribution and assume a uniform prior on $S$, which is the maximum entropy prior for $P_{r}(S)$. The posterior probability of success then follows a beta distribution, $P_{r}(S \mid D) \sim \operatorname{Bet} a(x+1, n-x+1)$. To quantify the uncertainty in the posterior probability of success estimates, the $95 \%$ posterior intervals can be computed numerically from the 2.5 and 97.5 percentiles of the beta inverse cumulative distribution function. We can then say that there is a $95 \%$ chance that the true probability of success lies in these intervals.

$N=3000$ mission scenarios (Monte Carlo runs) were run using our BERS simulator. From their starting locations, the GRU sense and move to any fires within its sensor radius, and deploy water on any fires within its water range. The fires are also moving and spreading towards the DA location. When there are multiple fire locations, the GRU are programmed to deploy water on fires that are closest to the DA location to protect the asset. In the set of binary data, $D=1$ if a fire came within 1 cell of the DA location and $D=0$ otherwise. From $D$, the probability of survival for the DA was determined over the range of starting distances between the DA and GRU, and the initial number of GRU.

Fig. 5 (left) shows the probability of the DA surviving given the starting distance between the DA and GRU and the number of GRU. There was a high probability $(>0.9)$ of DA survival given an initial number of 5 or more GRU and a starting distance between GRU and DA of approximately 3 to 15 cells. Fig. 5 (right) shows the probability of the DA surviving given the starting distance between the DA and GRU and for the 6 GRU case. An increasing probability from 0 to 15 cells is likely due to GRU being destroyed when located too close to the DA. The probability is seen to decrease suddenly at a distance of 20 cells. This is possibly due to GRU 
near the maximum of their water or sensing ranges (which was 20 cells). The maxima at 35-40 cells warrants further investigation. Generally, the probability of DA survival is seen to reduce as GRU are initially located further away.

For a different measure of uncertainty, the maximum standard error was calculated to be 0.11 for Fig. 5 (left) and 0.08 for Fig. 5 (right). The magnitude of the maximum standard errors indicates that the variations of probability seen over the initial GRU-DA distances are genuine and not artifacts of the simulation environment. A challenge in UQ is that some measures of uncertainty are open to be misinterpreted (Barde and Barde (2012)). The best approach to UQ that minimizes misinterpretation will be explored in future analysis.
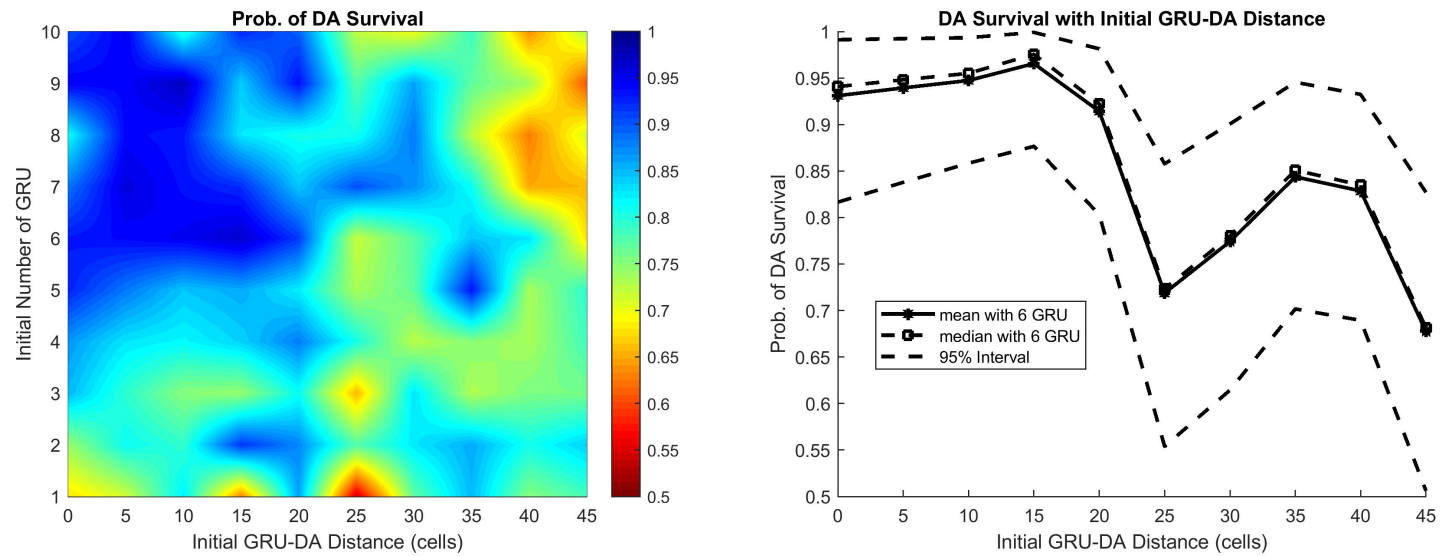

Figure 5. Left: Probability of the DA surviving given the starting distance between DA and GRU and the initial number of GRU (Maximum Standard Error is 0.11). Right: Probability of the DA surviving given the starting distance between DA and GRU and a team of 6 GRU (Maximum Standard Error is 0.08).

The probability of survival for the GRU was determined over the range of starting distances between the BFT and GRU, and the initial number of GRU. Fig. 6 (left) shows the probability of one GRU surviving given an initial number of GRU. It can be observed that the probability of one GRU surviving increases near linearly until 6 GRU, where it appears to saturate at a probability of approximately 0.8 as the effect of increasing the size of the team increases the risk of losing a GRU. Fig. 6 (right) shows the probability of one GRU surviving given the starting distance between GRU and BFT and a team of 6 GRU. This information can be crucial for the design of a response task force, and also tactical operational decisions.
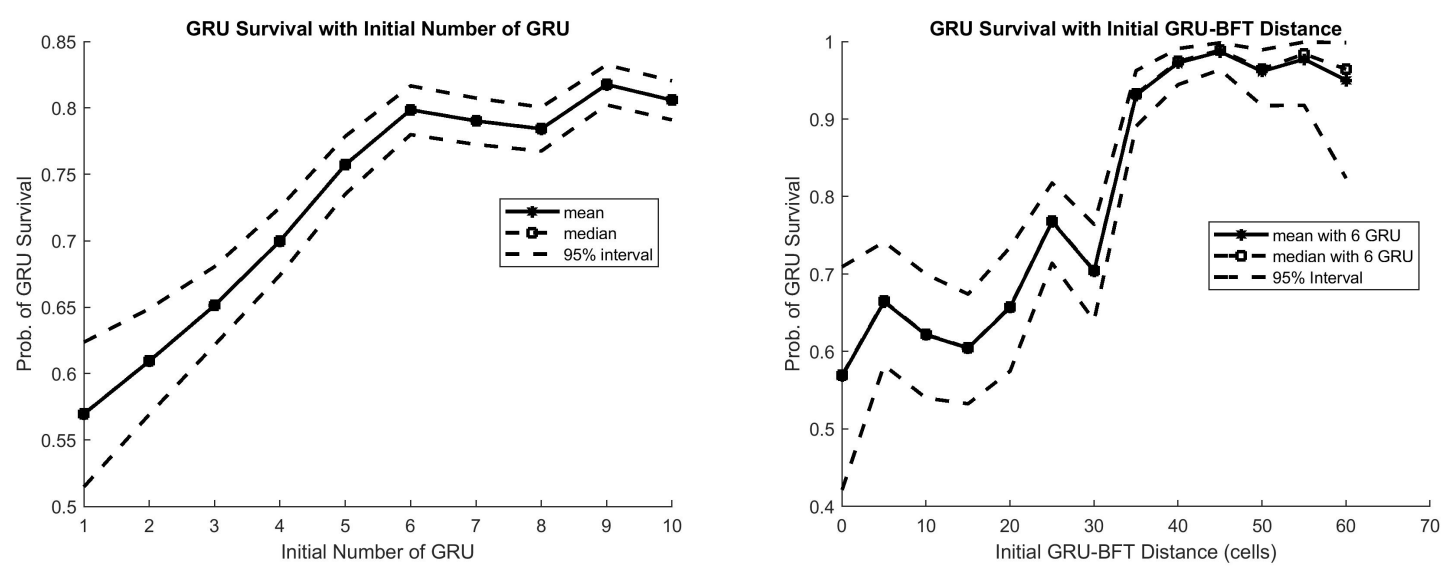

Figure 6. Left: Probability of one GRU surviving given the initial number of GRU (Maximum Standard Error is 0.11). Right: Probability of one GRU surviving given the starting distance between GRU and BFT and a team of 6 GRU (Maximum Standard Error is 0.03). 


\section{CONCLUSION}

In bushfire emergency response there are many different types of uncertainty to be quantified including, but not limited to, uncertainty around the mission, objectives and human behaviour. This paper only considers a few types of uncertainty, ones that could be easily quantified using standard computer-based simulation and Monte Carlo methods. Future work will consider more sources of uncertainty as well as communication of that uncertainty to decision makers. These results can inform decision making and policy regarding matters such as force design, C2 strategies, sensing, fire-fighting equipment, response unit type and equipage. Additionally, a future activity of this project is to adapt these UQ methodologies and apply them to specific Defence problems, to assess their broad applicability.

\section{ACKNOWLEDGEMENT}

This research was performed in collaboration with the Defence Science and Technology Group. We thank Mr Edward Dawson for his helpful advice in the simulator design and facilitation of this research.

\section{REFERENCES}

Barde, M. P. and P. J. Barde (2012). What to use to express the variability of data: Standard deviation or standard error of mean? Perspectives in clinical research 3(3), 113.

Bolstad, W. M. (2010). Understanding Computational Bayesian Statistics. Wiley Series in Computational Statistics. Wiley.

Bruggemann, T., J. J. Ford, G. White, and T. Perez (2019). Bushfire emergency response simulation. In The 23rd International Congress on Modelling and Simulation (MODSIM2019).

French, I., B. Cechet, T. Yang, and A. Sanabria (2013). FireDST: Fire impact and risk evaluation decision support toolmodel description. In 20th International Congress on Modelling and Simulation (MODSIM 2013), Adelaide, December.

Freni, G. and G. Mannina (2010). Bayesian approach for uncertainty quantification in water quality modelling: The influence of prior distribution. Journal of Hydrology 392(1-2), 31-39.

Green, D. G. (1993). Emergent behaviour in biological systems. Complex systems: from biology to computation, 24-35.

Jaynes, E. T. (1957). Information theory and statistical mechanics. Physical review 106(4), 620.

Johnston, P., J. Kelso, and G. J. Milne (2008). Efficient simulation of wildfire spread on an irregular grid. International Journal of Wildland Fire 17(5), 614-627.

Kallinen, V., T. Perez, T. Bruggemann, J. Ford, et al. (2019). A case study in uncertainty quantification of UAS behaviours against operational requirements. In AIAC18: 18th Australian International Aerospace Congress (2019), pp. 356. Engineers Australia, Royal Aeronautical Society.

Kapur, J. N. (1989). Maximum-entropy models in science and engineering. John Wiley \& Sons.

Kroese, D. P., T. Taimire, and Z. I. Botev (Eds.) (2011). Handbook of Monte Carlo Methods. Wiley Series in Probability and Statistics. Wiley.

Miller, C., J. Hilton, A. Sullivan, and M. Prakash (2015). SPARK-a bushfire spread prediction tool. In International Symposium on Environmental Software Systems, pp. 262-271. Springer.

Perez, T. (2015). Ship seakeeping operability, motion control, and autonomy-a bayesian perspective. IFACPapersOnLine 48(16), 217-222.

Perez, T., B. Williams, and P. de Lamberterie (2012). Computational aspects of probabilistic assessment of UAS robust autonomy. In Proceedings of the 28th Congress of the International Council of the Aeronautical Sciences, pp. 1-9. International Council of the Aeronautical Sciences.

Smith, R. C. (2013). Uncertainty quantification: theory, implementation, and applications, Volume 12. Siam.

Sullivan, A. L. (2009). Wildland surface fire spread modelling, 1990-2007. 3: Simulation and mathematical analogue models. International Journal of Wildland Fire 18(4), 387-403.

Tolhurst, K., B. Shields, D. Chong, et al. (2008). Phoenix: development and application of a bushfire risk management tool. Australian Journal of Emergency Management, The 23(4), 47.

Twomey, B. and A. Sturgess (2016). Simulation analysis-based risk evaluation (SABRE) fire: operational stochastic fire spread decision support capability in the queensland fire and emergency service. Proceedings of AFAC 2016.

Willems, J. C. and J. W. Polderman (2013). Introduction to mathematical systems theory: a behavioral approach, Volume 26. Springer Science \& Business Media. 